October 2017, Volume 2, Issue 4 (73-75)

\title{
The Assessment of Left Ventricular Function in MRI Using the Detection of Myocardial Borders and Optical Flow Approaches: A Review
}

\author{
Narjes Benameur ${ }^{1,}$, Younes Arous ${ }^{2,3}$, Nejmeddine Ben \\ Abdallah ${ }^{2,3}$, Tarek Kraiem ${ }^{1,3}$ \\ ${ }^{1} \mathrm{PhD}$, Laboratory of Biophysics and Medical Technologies, Higher Institute of \\ Medical Technologies of Tunis, University of Tunis El Manar, Tunis, Tunisia \\ ${ }^{2}$ Military Hospital of Instruction of Tunis, Tunisia \\ ${ }^{3}$ University of Tunis El Manar, Faculty of Medicine, Tunis, Tunisia \\ ${ }^{*}$ Corresponding author: Narjes Benameur, PhD, laboratory of Biophysics and \\ Medical Technologies, Higher Institute of Medical Technologies of Tunis, University \\ of Tunis El Manar,Tunis, Tunisia.E-mail:narjes.benameur@yahoo.fr
}

DOI: $10.21859 /$ ijcp-030101

Submited: 05.19.2017

Accepted: 07.01.2017

Keywords:

Myocardial Borders

Segmentation

Cardiac MRI

Flow Cytometry

Regional Dysfunction

(C) 2017. International Journal of Cardiovascular Practice.

\begin{abstract}
The evaluation of left ventricular wall motion in Magnetic Resonance Imaging (MRI) clinical practice is based on a visual assessment of cine-MRI sequences. In fact, clinical interpreters (radiologists) proceed with a global visual evaluation of multiple cine-MRI sequences acquired in the three standard views. In addition, some functional parameters are quantified following a manual or a semi-automatic contouring of the myocardial borders. Although these parameters give information about the functional state of the left ventricle, they are not able to provide the location and the extent of wall motion abnormalities, which are associated with many cardiovascular diseases. In the past years, several approaches were developed to overcome the limitations of the classical evaluation techniques of left ventricular function. The aim of this article is to present an overview of the different methods and to summarize the relevant techniques based on myocardial contour detection and optical flow for regional assessment of left ventricular abnormalities.
\end{abstract}

\section{INTRODUCTION}

In recent years, several researches have focused on the study of the regional left ventricle (LV) function in cardiac Magnetic Resonance Imaging (MRI) [1-3]. Despite the multiplicity of methods and algorithms proposed in the literature, their main topic remains the development of precise quantitative parameters and the combination of information collected by the use of different modalities in order to assist in left ventricular diagnosis. These methods can be classified into three main families: the first family includes all the methods dedicated to the detection of myocardial borders [4-6]. The second family is relative to cardiac deformation monitoring approaches based on optical flow methods $[7,8]$ third family entails the techniques of computing parametric images used to study the regional cardiac function [9-12]. In this article, we intend to present an overview of the two first approaches: the detection of myocardial borders and optical flow methods.

\section{Approaches Bases on the Detection of Myocardial Borders}

The study of left ventricular function in cardiac imaging, specifically in MRI, requires a delineation of the myocardial contours in order to have access to global parameters such as systolic ejection volume, myocardial mass, and left ventricular ejection fraction (LVEF). Usually, this segmentation is established manually by the radiologist or semi-automatically with some adjustments made by the manipulator $[13,14]$. The result of LVEF depends on the specificity and the quality of the delineation of the endocardial and epicardial contours. This procedure is considered a very difficult task in practice since it requires the segmentation of the myocardial edges through a series of images in short-axis view. In clinical practice, some radiologists use only the myocardial contours of the end diastole image (corresponding to the telediastolic moment when the left ventricular volume reaches its maximum) and the end systole image (where the volume of the LV is at a minimum) to compute LVEF. To overcome these limitations, several researchers have developed semi-automatic or automatic approaches for the detection of myocardial contours [4, 15]. We distinguish the approaches based on the intensity of the pixels which were initially proposed for the segmentation of the endocardial contours. These techniques such as histogram thresholding methods and their derivatives (adaptive thresholding methods) make use of the distribution of gray levels in the image to separate the ventricular cavity from the rest of the 
objects in this image $[16,17]$. The results of these techniques are very dependent on the contrast of the image and the choice of the threshold value. Moreover, these approaches do not take into account the geometric shape of the object to be detected, making the task of segmentation very difficult especially with regard to the shape of the cardiac cavities.

Other segmentation methods, based on clustering, have been developed in the literature $[18,19]$. Among the most used approaches belonging to this family are those based on the K-means algorithm and other classification methods [20, 21]. The latter attributes some specific characteristics for each pixel of the image, resulting in a set of different classes. Each class contains pixels with similar properties. The image is then divided into sub-regions, making it possible to segment the object of interest. These methods have proven their accuracy in detecting objects in medical images. However, their robustness in cardiac MRI is constrained by the irregular shape of the myocardial contours and the interdependence of the pixels with their neighborhood. The limitations inherent to the previous methods are relatively overcome by the advent of new approaches described as "high level" approaches. The algorithms relative to these techniques integrate geometric models that are well adapted to the topology and the irregularities of myocardial borders thanks to an iterative process including a priori knowledge. Among the techniques based on this approach, we can name the parametric deformable models like the active contour methods or "Snakes" and the geometric models such as the level set methods [22-24]. These models are most adapted to the segmentation of endocardial and epicardial contours and are based on the minimization of an energy function after placing an initial curve close to the contour of the myocardium to be detected. In an iterative process, this curve gradually moves towards the object of interest until it coincides with the desired contour. The forces which push the initialization curve to deform and to move towards the desired object are associated with an energy function which takes into account the characteristics of the curve (internal forces), the properties of the image (External forces), and the interaction between the curve and the image $[25,26]$. Like the active contour methods, the "level set" algorithms require an initialization of a curve that must be represented implicitly. This contour is considered as the level 0 of a scalar function that must be of a higher dimension. This curve evolves at a speed that is dependent on time and on the characteristics of this curve (the mean curvature or the normal curvature). The level set method allows a good adaptation to the irregularities of the myocardial contours with more precision in the detection. However, the methods based on the deformable model, whether parametric or geometric models, require a user interaction and they are very sensitive to the initialization step $[4,27]$. In recent years, new hybrid methods using deformable model algorithms combined with statistical models have yielded promising results in the detection of left ventricular abnormalities. These methods include the Active Appearance model (AAM) and the Point Distribution Model (PDM) [28, 29].

\section{Optical Flow Methods}

Several studies based on the optical flow approach have been developed in the literature to estimate the movement and the displacement of the myocardium $[30,31]$. This approach is a representation of the apparent movement of objects from a sequence of images. It relies mainly on the assumption of the invariance of the intensities of pixels over time and on the spatial coherence. Respecting these assumptions is crucial to estimate the movement that is usually measured between two consecutive images. However, several authors have used more images in their work to increase the accuracy and the stability of estimation. There are many methods of optical flow that can be classified into three families: differential optical flow, optical flow based on energy, and other algorithms based on phase. Among the works on differential optical flow, we can name those of Horn and Schunck, which are among the first works on this approach [32]. Their method describes the apparent motion of an object by measuring for each pixel a velocity vector that describes its displacement between two successive images. The differential notation comes from the fact that the motion is estimated from the spatio- temporal derivatives of image intensities $[8,33]$. Other approaches based on energy have shown very promising results in cardiac MRI. They involve a frequency technique that uses a Gabor 3D filter bank to estimate the motion in a sequence of images $[34,35]$. This approach assumes that the tracking of the movement of an object over time requires the identification of the orientation of this object in a space-time repository, also called a space-time space. In this context, Heeger et al. [36] proposed the use of a set of Gabor 3D filters in their work on optical flow: each filter is designed to search for a well-defined frequency. The combination of the responses of these filters allows the calculation of the energies and the estimation of the optical flow for each pixel. From these energy-based methods, other approaches based on the phase were derived. These methods use Gabor 3D filters and the same general equations of the optical flow for the computation of phase features. However, unlike the differential methods based on the invariance of the intensity, these approaches rely on the invariance of the phase gradient over time for the estimation of the velocity of the displacement [37, 38]. The main limitations of these spatio-temporal approaches lie in the difficulty of respecting the optical flow assumptions and the relatively high number of used filters, making the estimation process very complex [39].

\section{CONCLUSIONS}

In the present article, we reviewed two different approaches for the assessment of left ventricular function. The first approach is based on the delineation of myocardial borders in order to provide a precise measurement of the LVEF. The second method described from literature is based on the optical flow algorithm. The validation of these two approaches in cine MRI and tagged sequences reveal that they seem to represent promising techniques that are likely to improve the detection of left ventricular wall motion abnormalities. However, further studies are needed to ameliorate the reproducibility of these techniques and facilitate their integration in cardiac MRI clinical routine.

\section{CONFLICTS OF INTEREST}

There is no conflicts of interest to declare.

\section{REFERENCES}

1. Attili AK, Schuster A, Nagel E, Reiber JH, van der Geest RJ. Quantification in cardiac MRI: advances in image acquisition and pro- 
cessing. Int J Cardiovasc Imaging. 2010;26 Suppl 1:27-40. DOI: 10.1007/s10554-009-9571-x PMID: 20058082

2. Alessandrini M, Basarab A, Liebgott H, Bernard O. Myocardial motion estimation from medical images using the monogenic signal. IEEE Trans Image Process. 2013;22(3):1084-95. DOI: 10.1109/ TIP.2012.2226903 PMID: 23193239

3. Afshin M, Ben Ayed I, Punithakumar K, Law M, Islam A, Goela A, et al. Regional assessment of cardiac left ventricular myocardial function via MRI statistical features. IEEE Trans Med Imaging. 2014;33(2):481-94. DOI: 10.1109/TMI.2013.2287793 PMID: $\underline{24184708}$

4. Santarelli MF, Positano V, Michelassi C, Lombardi M, Landini L. Automated cardiac MR image segmentation: theory and measurement evaluation. Med Eng Phys. 2003;25(2):149-59. PMID: 12538069

5. Tsadok Y, Petrank Y, Sarvari S, Edvardsen T, Adam D. Automatic segmentation of cardiac MRI cines validated for long axis views. Comput Med Imaging Graph. 2013;37(7-8):500-11. DOI: 10.1016/j. compmedimag.2013.09.002 PMID: 24094590

6. Piazzese C, Carminati MC, Colombo A, Krause R, Potse M, Auricchio A, et al. Segmentation of the left ventricular endocardium from magnetic resonance images by using different statistical shape models. J Electrocardiol. 2016;49(3):383-91. DOI: 10.1016/j.jelectrocard.2016.03.017 PMID: 27046100

7. Spies H, Barron J. Evaluating Certainties in Image Intensity Differentiation for Optical Flow. First Canadian Conference on Computer and Robot Vision2004. p. 408-16.

8. Torkashvand P, Behnam H, Sani ZA. Modified optical flow technique for cardiac motions analysis in echocardiography images. J Med Signals Sens. 2012;2(3):121-7. PMID: 23717803

9. Caiani EG, Toledo E, MacEneaney P, Bardo D, Cerutti S, Lang RM, et al. Automated interpretation of regional left ventricular wall motion from cardiac magnetic resonance images. J Cardiovasc Magn Reson. 2006;8(3):427-33. PMID: 16755828

10. Redheuil AB, Kachenoura N, Laporte R, Azarine A, Lyon X, Jolivet $\mathrm{O}$, et al. Interobserver variability in assessing segmental function can be reduced by combining visual analysis of CMR cine sequences with corresponding parametric images of myocardial contraction. J Cardiovasc Magn Reson. 2007;9(6):863-72. DOI: 10.1080/10976640701693626 PMID: 18066745

11. Rejab H, Kridis WB, Ben Ameur H, Feki J, Frikha M, Beyrouti MI. [Rectal stromal tumor: report of a case]. Pan Afr Med J. 2014;17:119. DOI: $10.11604 /$ pamj.2014.17.119.2285 PMID: 25120863

12. Benameur N, Caiani EG, Arous Y, Abdallah NB, Kraiem T. Interpretation of cardiac wall motion from cine-MRI combined with parametric imaging based on the Hilbert transform. MAGMA. 2017;30(4):347-57. DOI: 10.1007/s10334-017-0609-0 PMID: $\underline{28220266}$

13. Evin M, Cluzel P, Lamy J, Rosenbaum D, Kusmia S, Defrance C, et al. Assessment of left atrial function by MRI myocardial feature tracking. J Magn Reson Imaging. 2015;42(2):379-89. DOI: 10.1002/ imri.24851 PMID: 25630749

14. Barkhausen J, Ruehm SG, Goyen M, Buck T, Laub G, Debatin JF. MR evaluation of ventricular function: true fast imaging with steadystate precession versus fast low-angle shot cine MR imaging: feasibility study. Radiology. 2001;219(1):264-9. DOI: 10.1148/radiology.219.1.r01ap12264 PMID: 11274568

15. Bricq S, Frandon J, Bernard M, Guye M, Finas M, Marcadet L, et al. Semiautomatic detection of myocardial contours in order to investigate normal values of the left ventricular trabeculated mass using MRI. J Magn Reson Imaging. 2016;43(6):1398-406. DOI: 10.1002/ jmri.25113 PMID: 26646347

16. Lee HY, Codella N, Cham M, Prince M, Weinsaft J, Wang Y. Left ventricle segmentation using graph searching on intensity and gradient and a priori knowledge (lvGIGA) for short-axis cardiac magnetic resonance imaging. J Magn Reson Imaging. 2008;28(6):1393-401. DOI: $10.1002 /$ imri.21586 PMID: 19025947

17. Lynch M, Ghita O, Whelan PF. Automatic segmentation of the left ventricle cavity and myocardium in MRI data. Comput Biol Med. 2006;36(4):389-407. DOI: 10.1016/j.compbiomed.2005.01.005 PMID: 15925359

18. Lalande A, Legrand L, Walker PM, Jaulent MC, Guy F, Cottin Y et al. Automatic detection of cardiac contours on MR images using fuzzy logic and dynamic programming. Proc AMIA Annu Fall Symp. 1997:474-8. PMID: 9357671

19. Boudraa Ae-O. Automated detection of the left ventricular region in magnetic resonance images by Fuzzy c-Means model. Int J Card Imaging. 1997;13(4):347-55. PMID: 9306149

20. Lee H. Segmentation with K-means Clustering and Graph Searching on Cardiac MRI. Int J Med Health Biomed Bioeng Pharm Eng. 2015;9:292-5.

21. Debreuve E, Barlaud M, Aubert G, Laurette I, Darcourt J. Space-time segmentation using level set active contours applied to myocardial gated SPECT. IEEE Trans Med Imaging. 2001;20(7):643-59. DOI: 10.1109/42.932748 PMID: 11465470

22. Malladi R, Sethian JA, Vemuri BC. Shape modeling with front propagation: a level set approach. IEEE Transact Pattern Anal Mach Intel. 1995;17(2):158-75. DOI: $10.1109 / 34.368173$

23. Lee HY, Codella NC, Cham MD, Weinsaft JW, Wang Y. Automatic left ventricle segmentation using iterative thresholding and an active contour model with adaptation on short-axis cardiac MRI. IEEE Trans Biomed Eng. 2010;57(4):905-13. DOI: 10.1109/ TBME.2009.2014545 PMID: 19203875

24. Cho J, Benkeser PJ. Cardiac segmentation by a velocity-aided active contour model. Comput Med Imaging Graph. 2006;30(1):31-41. DOI: 10.1016/j.compmedimag.2005.10.006 PMID: 16378714

25. Peng P, Lekadir K, Gooya A, Shao L, Petersen SE, Frangi AF. A review of heart chamber segmentation for structural and functional analysis using cardiac magnetic resonance imaging. MAGMA. 2016;29(2):155-95. DOI: 10.1007/s10334-015-0521-4 PMID: 26811173

26. Hautvast G, Lobregt S, Breeuwer M, Gerritsen F. Automatic contour propagation in cine cardiac magnetic resonance images. IEEE Trans Med Imaging. 2006;25(11):1472-82. DOI: 10.1109/ TMI.2006.882124 PMID: 17117776

27. Corsi C, Lamberti C, Catalano O, MacEneaney P, Bardo D, Lang $\mathrm{RM}$, et al. Improved quantification of left ventricular volumes and mass based on endocardial and epicardial surface detection from cardiac MR images using level set models. J Cardiovasc Magn Reson. 2005;7(3):595-602. PMID: 15959973

28. van der Geest RJ, Lelieveldt BP, Angelie E, Danilouchkine M, Swingen $\mathrm{C}$, Sonka $\mathrm{M}$, et al. Evaluation of a new method for automated detection of left ventricular boundaries in time series of magnetic resonance images using an Active Appearance Motion Model. J Cardiovasc Magn Reson. 2004;6(3):609-17. PMID: 15347125

29. Kaus MR, von Berg J, Weese J, Niessen W, Pekar V. Automated segmentation of the left ventricle in cardiac MRI. Med Image Anal. 2004;8(3):245-54. DOI: 10.1016/j.media.2004.06.015 PMID: 15450219

30. Angelini ED, Gerard O. Review of Myocardial Motion Estimation Methods from Optical Flow Tracking on Ultrasound Data. 28th Annual International Conference of the IEEE2006. p. 1537-40.

31. Ibarra E, Medina R, Morocho V, Vanegas P. Optical Flow as a Tool for Cardiac Motion Estimation. Asia-Pacific Conference on Computer Aided System Engineering2015. p. 173-8.

32. Horn BKP, Schunck BG. Determining optical flow. Artif Intel. 1981;17(1-3):185-203. DOI: 10.1016/0004-3702(81)90024-2

33. Spies H, Barron J. Evaluating Certainties in Image Intensity Differentiation for Optical Flow. First Canadian Conference on Computer and Robot Vision; Canada2004.

34. Sundar H, Litt H, Shen D. Estimating Myocardial Motion by 4D Image Warping. Pattern Recognit. 2009;42(11):2514-26. DOI: 10.1016/j.patcog.2009.04.022 PMID: 20379351

35. Jia K, Xiaogang W, Xiaoou T. Optical flow estimation using learned sparse model. IEEE International Conference on Computer Vision (ICCV); Barcelona, Spain2011. p. 2391-8.

36. Heeger DJ. Optical flow using spatiotemporal filters. Int J Comput Vis. 1988;1(4):279-302. DOI: $10.1007 /$ bf00133568

37. Wang L, Basarab A, Girard PR, Croisille P, Clarysse P, Delachartre P. Analytic signal phase-based myocardial motion estimation in tagged MRI sequences by a bilinear model and motion compensation. Med Image Anal. 2015;24(1):149-62. DOI: 10.1016/i.media.2015.06.005 PMID: 26176412

38. Xavier M, Lalande A, Walker PM, Brunotte F, Legrand L. An adapted optical flow algorithm for robust quantification of cardiac wall motion from standard cine-MR examinations. IEEE Trans Inf Technol Biomed. 2012;16(5):859-68. DOI: 10.1109/TITB.2012.2204893 PMID: 22717523

39. Barron JL, Fleet DJ, Beauchemin SS. Performance of optical flow techniques. Int J Comput Vis. 1994;12(1):43-77. DOI: 10.1007/ bf01420984 